\title{
Análisis de susceptibilidad antimicrobiana y hallazgos microbiológicos: perfil en cirugía abdominal de urgencia
}

\author{
Katya Carrillo ${ }^{1}$, Sebastián López ${ }^{1}$, Günther Bocic ${ }^{1}$, Mario Luppi², \\ Fernanda Ávila ${ }^{2}$, Roberto Olivares ${ }^{2}$, Felipe Bocic ${ }^{3}$, Mario Abedrapo ${ }^{1}$, \\ Rodrigo Azolas ${ }^{1}$, Antonella Sanguineti ${ }^{1}$, Mauricio Díaz ${ }^{1}$ y José Luis Llanos ${ }^{1}$
}

\section{Microbiological findings and antimicrobial susceptibility analysis: emergency general abdominal surgery profile}

Background: Antimicrobial resistance is a worldwide problem. In patients requiring emergency surgery, antibiotics are an important assistance additional to surgical intervention. Objective: Analize peritoneal fluid cultures obtaines from patients who underwent emergency surgery. Methods: A prospective cohort of emergency abdominal surgical patients were enrolled. Peritoneal fluid cultures were taken and processed according to standarized technique. Results: A 39.4\% of positive cultures was found. E. coli was the most common bacteria identified. Five positive cultures for P. aeruginosa stand out. E. coli had $25 \%$ resistance to ampicillin/sulbactam and 19\% for quinolones. Conclusion: Resistance found was lower than international reports, but still noteworthy. Knowledge of local bacteria profile and antimicrobial resistance is important for local antibiotic hospital policy.

Key words: Peritoneal fluid; Emergency surgery; Antibiogram.

\section{Resumen}

Introducción: La resistencia a antibióticos es un problema mundial. En los pacientes que requieren cirugía de urgencia, los antibióticos son un apoyo importante concomitante al acto quirúrgico. Objetivo: Analizar los cultivos de líquido peritoneal obtenidos de pacientes operados por patología quirúrgica abdominal de urgencia. Materiales y Métodos: Se realiza una cohorte prospectiva de los pacientes operados de urgencia. Se tomó cultivo de líquido peritoneal y se procesó según técnica estandarizada. Resultados: Se encontró un $39,4 \%$ de cultivos positivos. E. coli fue el germen más frecuente. Destacan 5 cultivos positivos para $P$. aeruginosa. Existe un $25 \%$ de resistencia a ampicilina/sulbactam y $19 \%$ a quinolonas para $E$. coli. Conclusión: La resistencia encontrada fue menor a lo reportado en la literatura, pero aún destacable. El conocimiento del perfil de bacterias y sus resistencias a antimicrobianos son importantes para las políticas hospitalarias locales de uso racional de antibióticos.

Palabras clave: Líquido peritoneal; Cirugía urgencia; Antibiograma.

\section{Introducción}

Desde el descubrimiento de la penicilina por Alexander Fleming en 1928, el uso de antibióticos ha revolucionado el manejo de la infecciones, tanto en el ámbito quirúrgico como en el no quirúrgico. Sin embargo, actualmente existe un problema a nivel mundial dado por la resistencia progresiva de las bacterias a los antibióticos más frecuentemente utilizados, determinando así la necesidad de una constante búsqueda y descubrimiento de nuevos antibióticos para el tratamiento de estas cepas ${ }^{1}$. La resistencia a antimicrobianos constituye un problema objetivo y real en la atención de salud, a nivel mundial y local ${ }^{2}$. Es importante señalar que el área quirúrgica no es ajena a esta situación. Por ello, diversas entidades han enfatizado la necesidad de implementar programas de vigilancia y de inter-
'Departamento de Cirugía ¿Unidad de Infectología, Departamento de Medicina Interna.

${ }^{3}$ Interno de Medicina Universidad de Chile. Hospital Clínico de la Universidad de Chile, Santiago, Chile.

Recibido el 4 de mayo de 2017, aceptado para publicación el 29 de junio de 2017

Correspondencia a:

Günther Bocic

guntherbocic@hotmail.com 
vención, que puedan reflejar una disminución en la morbimortalidad y costos asociados a la resistencia a antimicrobianos ${ }^{2-5}$.

En la práctica quirúrgica, ésta es una problemática permanente que requiere de la intervención multidisciplinaria de todos los miembros de los equipos de infecciones nosocomiales.

Las infecciones intraabdominales (IIA) son muy frecuentes y se producen casi siempre por perforación o inflamación de la pared intestinal u otras vísceras huecas, especialmente a partir de la flora gastrointestinal. El diagnóstico microbiológico de la mayoría de las IIA es directo y se fundamenta en el cultivo de muestras representativas de los diversos procesos patológicos ${ }^{6}$. Lo anterior debería ser factible por medio de una adecuada vigilancia epidemiológica, con la obtención de datos cuantitativos de resistencia en microorganismos relevantes, ya sea por su prevalencia y/o por sus mecanismos de resistencia. Con esto se debe favorecer el diseño de estrategias orientadas a lograr el uso racional de los antimicrobianos, evitando la emergencia de nuevas resistencias y su posterior amplificación ${ }^{2}$. La recolección de información debería ser seriada y continua en el tiempo, logrando observar la evolución de susceptibilidades microbiológicas en los distintos contextos hospitalarios y realidades locales.

Es en este contexto es que se decide realizar un estudio de cohorte prospectiva, con la revisión prospectiva de datos microbiológicos de cultivo de líquido peritoneal en un grupo de pacientes nuevos, admitidos por alguna patología quirúrgica abdominal de urgencia para evaluar cepas y susceptibilidades actuales.

Tabla 1. Características generales (por paciente)

\begin{tabular}{|lccc|}
\hline & $\begin{array}{c}\text { Total (\%) } \\
\mathbf{n = 1 4 2}\end{array}$ & $\begin{array}{c}\text { Hombres (\%) } \\
\mathbf{n}=\mathbf{7 1}\end{array}$ & $\begin{array}{c}\text { Mujeres (\%) } \\
\mathbf{n}=\mathbf{7 1}\end{array}$ \\
\hline Edad (años) & $46,9 \pm 20,9$ & $47,3 \pm 20,3$ & $46,6 \pm 21,7$ \\
Cultivo positivo & $56(39,4 \%)$ & $34(47,9 \%)$ & $22(31 \%)$ \\
$\quad>1$ microorganismo & 11 & 7 & 4 \\
Diagnóstico & & & \\
$\quad$ Apendicitis aguda & $74(52,1 \%)$ & $38(53,5 \%)$ & $36(50,7 \%)$ \\
Colecistitis aguda & $30(21,1 \%)$ & $15(21,1 \%)$ & $15(21,1 \%)$ \\
Obstrucción intestinal & $12(8,5 \%)$ & $7(9,9 \%)$ & $5(7 \%)$ \\
Perforación víscera hueca & $9(6,3 \%)$ & $6(8,5 \%)$ & $3(4,2 \%)$ \\
Hernias & $7(4,9 \%)$ & $2(2,8 \%)$ & $5(7 \%)$ \\
Otros & $10(7 \%)$ & $3(4,2 \%)$ & $7(9,9 \%)$ \\
\hline
\end{tabular}

\section{Materiales y Métodos}

El análisis de una cohorte prospectiva se realizó desde abril a diciembre de 2015, en todos los pacientes ingresados al Servicio de Urgencia de Hospital Clínico de la Universidad de Chile con alguna patología abdominal que motivó una intervención quirúrgica de urgencia. Dentro de los criterios de inclusión, se consideraron a todos los pacientes adultos mayores de 15 años, que consultaron por algún cuadro de etiología quirúrgica. Se excluyó a pacientes con hospitalizaciones recientes (definidas dentro de los últimos 3 meses), inmunosuprimidos y que se encontraran recibiendo antibióticos por cualquier causa al momento de la cirugía.

Se obtuvo muestras de líquido peritoneal, cuando estuvo presente, para cultivo corriente según técnica estandarizada. En caso contrario, se obtuvo muestra de lavado con solución fisiológico del sitio quirúrgico. Independiente de la indicación de cirugía, se tomaron muestras de líquido peritoneal para cultivo corriente, que fueron procesadas según los estándares habituales y normativa vigente local. Se utilizaron medios de Agar-Sangre, MacConkey y Tioglicolato, y metodología automatizada ( $\mathrm{Hi}$ Tech), no se incluyó otros cultivos especiales (hongos, etc.).

Posteriormente, se rescataron y tabularon los resultados microbiológicos, gérmenes involucrados y sus susceptibilidades en el antibiograma. Se realizó un análisis estadístico descriptivo de los datos obtenidos, consignando aspectos demográficos y epidemiológicos rescatados de la ficha electrónica de los pacientes.

\section{Resultados}

Se analizaron muestras de un total de 142 pacientes, $71(50 \%)$ de los cuales eran de sexo femenino y $71(50 \%)$ masculino. Las características demográficas de la población estudiada se resumen en la Tabla 1. Los cultivos de líquido peritoneal fueron satisfactoriamente positivos en 56 casos $(39,4 \%)$, de los cuales 11 fueron positivos para más de un microorganismo, y cultivos negativos en 86 casos $(60,6 \%)$. La distribución de cultivos positivos según variables demográficas y diagnósticos que motivaron la intervención quirúrgica y que permitieron la toma de cultivos de líquido peritoneal se resumen en la Tabla 2. Como es de esperar, la patología quirúrgica de urgencia en contexto de cuadros apendiculares y biliares fueron los más frecuentes. La tasa más alta de cultivos positivos fue en los pacientes operados 
por perforación de víscera hueca, en segundo lugar la apendicitis aguda y la menor tasa estuvo en el grupo operado por colecistitis aguda.

Del total de cultivos positivos, el $85,7 \%$ corresponde a enterobacterias con 7 géneros diferentes, la frecuencia de los gérmenes aislados se resume en la Figura 1. El microorganismo más frecuentemente aislado fue la Escherichia coli, en 37 de 154 cultivos analizados. En la Tabla 3, se muestra la susceptibilidad de E. coli in vitro, destacando una susceptibilidad de $75 \%$ para Ampicilina/Sulbactam y de $81 \%$ para Ciprofloxacino. En las Tablas 4 y 5 se muestran los microorganismos más frecuentes luego de E. coli. En 5 pacientes se identifica la presencia de Pseudomonas aeruginosa en el cultivo del líquido peritoneal. Se observa que $P$. aeruginosa presenta susceptibilidad completa a todo el pool de antimicrobianos in vitro. Respecto a Klebsiella pneumoniae, destaca susceptibilidad disminuida a Ampicilina/Sulbactam de un 66,7\%.
Tabla 2. Incidencia de cultivo positivo según sexo, edad y diagnóstico de ingreso (por paciente)

\begin{tabular}{|lcc|}
\hline & $\begin{array}{c}\text { Cultivo (+) } \\
\mathbf{n = 5 6}\end{array}$ & $\begin{array}{c}\text { Cultivo (-) } \\
\mathbf{n = 8 6}\end{array}$ \\
Sexo & $34(52,1 \%)$ & $37(47,9 \%)$ \\
$\quad$ Masculino & $22(31 \%)$ & $49(69 \%)$ \\
Femenino & $51,4 \pm 21,9$ & $44 \pm 19,9$ \\
Edad (años) & & \\
Diagnóstico & $35(47,3 \%)$ & $39(52,7 \%)$ \\
Apendicitis aguda & $6(20 \%)$ & $24(80 \%)$ \\
Colecistitis aguda & $3(25 \%)$ & $9(75 \%)$ \\
Obstrucción intestinal & $5(55,6 \%)$ & $4(44,4 \%)$ \\
Perforación víscera hueca & $2(28,6 \%)$ & $5(71,4 \%)$ \\
Hernias & $5(50 \%)$ & $5(50 \%)$ \\
Otros & & \\
\hline
\end{tabular}

Tabla 3. Susceptibilidad in vitro de Escherichia coli en población adulta con patología quirúrgica abdominal de urgencia

\begin{tabular}{|lc|}
\hline Antimicrobiano & $\begin{array}{c}\text { Cultivo peritoneal } \\
\text { (N: 37) } \\
\text { \%o }\end{array}$ \\
Gentamicina & 100 \\
Amikacina & 100 \\
Ciprofloxacino & 81 \\
Ampicilina/Sulbactam & 75 \\
Cotrimoxazol & 89 \\
Imipenem & 100 \\
Cefazolina & 92 \\
Cefotaxima & 95 \\
Piperacilina/Tazobactam & 97 \\
Meropenem & 100 \\
\hline
\end{tabular}

Los datos están expresados en porcentajes de susceptibilidad.
Tabla 4. Susceptibilidad in vitro de Pseudomonas aeruginosa en población adulta con patología quirúrgica abdominal de urgencia

\begin{tabular}{|lc|}
\hline Antimicrobiano & $\begin{array}{c}\text { Cultivo peritoneal } \\
(\mathbf{N}: \mathbf{5}) \\
\mathbf{\%}\end{array}$ \\
Gentamicina & 100 \\
Amikacina & 100 \\
Ciprofloxacino & 100 \\
Ampicilina/Sulbactam & - \\
Cotrimoxazol & - \\
Imipenem & 100 \\
Cefazolina & - \\
Cefotaxima & - \\
Piperacilina/Tazobactam & 100 \\
Meropenem & 100 \\
Ceftazidima & 100 \\
\hline Colistin & 100 \\
\hline
\end{tabular}

Los datos están expresados en porcentajes de cepas susceptibles.

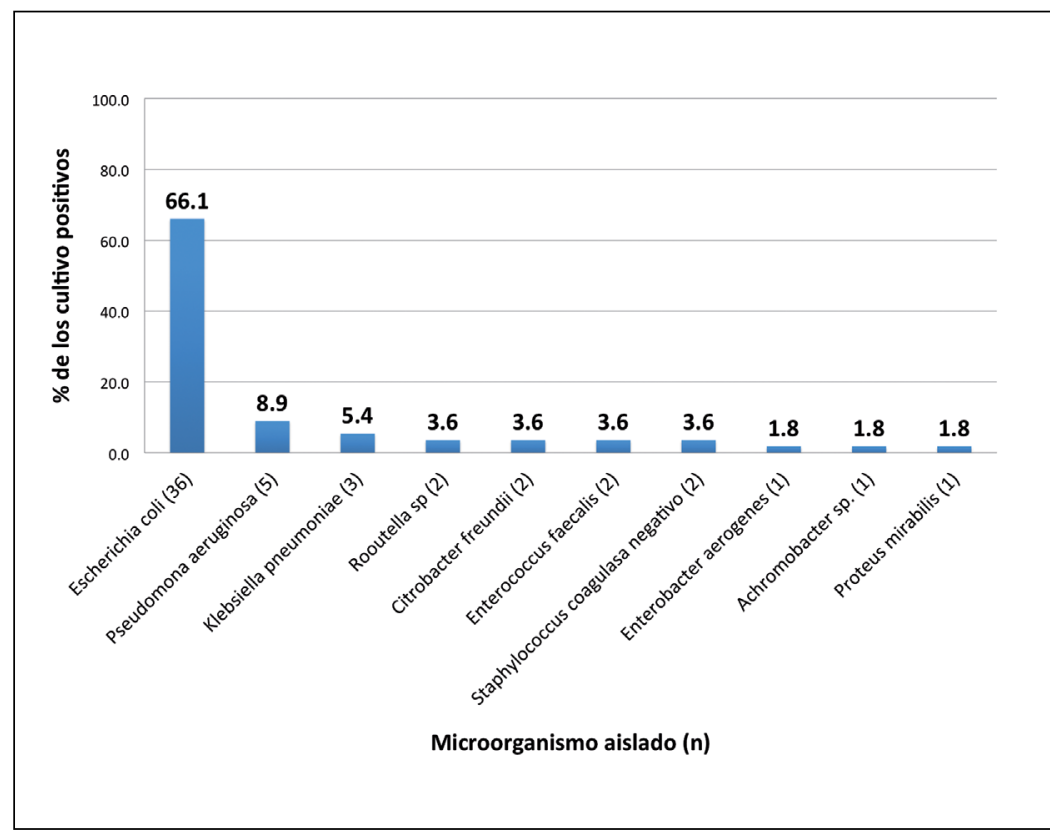

Figura 1. Frecuencia de microorganismos aislados en 56 cultivos positivos. 
Tabla 5. Susceptibilidad in vitro de Klebsiella pneumoniae en población adulta con patología quirúrgica abdominal de urgencia

\begin{tabular}{|lc|}
\hline Antimicrobiano & $\begin{array}{c}\text { Cultivo peritoneal } \\
\text { (N: 3) } \\
\text { \% }\end{array}$ \\
\hline Gentamicina & 100 \\
\hline Amikacina & 100 \\
Ciprofloxacino & 100 \\
Ampicilina/Sulbactam & 66,7 \\
\hline Cotrimoxazol & 100 \\
\hline Imipenem & 100 \\
\hline Cefazolina & 100 \\
\hline Cefotaxima & 100 \\
\hline Piperacilina/Tazobactam & 100 \\
\hline Meropenem & 100 \\
\hline Ertapenem & 100 \\
\hline
\end{tabular}

Los datos están expresados en porcentajes de cepas susceptibles.

En la Tabla 6, se muestran las otras cepas aisladas que, en su conjunto, representan cerca del 19,6\% de los cultivos positivos. Destaca que la resistencia registrada en los estudios de susceptibilidad por antibiograma para cada una de ellas es, en su mayoría, de muy bajo porcentaje.

\section{Discusión}

En el terreno quirúrgico y abdominal de urgencia, las infecciones suelen ser polimicrobianas y toma especial interés el manejo de las infecciones por bacterias gram negativas frecuentes en la flora intestinal. El uso apropiado de antibióticos, habitualmente empíricos, en este escenario ha demostrado disminuir la morbimortalidad ${ }^{7}$. Entre los focos de mayor interés a nivel mundial se encuentra la resistencia progresiva de enterobacterias, por mecanismos como la producción de betalactamasas de espectro extendido y carbapenemasas, así como la resistencia a fluoroquinolonas que son ampliamente utilizadas en el tratamiento de infecciones tanto gastrointestinales como del tracto urinario. En consecuencia, ha surgido un especial interés en la vigilancia constante de la susceptibilidad antimicrobiana de la realidad local en varios países. Iniciativas globales como SMART (Study for Monitoring Antimicrobial Resistance Trends), en la que participan cerca de 54 países, se han creado con este objetivo ${ }^{8-12}$.
Tabla 6. Otras cepas aisladas y cultivos peritoneales positivos

\begin{tabular}{|ll|}
\hline Microorganismo & n \\
\hline Rooutella sp & 2 \\
\hline Citrobacter freundii & 2 \\
\hline Enterococcus faecalis & 2 \\
\hline Staphylococcus coagulasa negativo & 2 \\
\hline Enterobacter aerogenes & 1 \\
Achromobacter sp. & 1 \\
\hline Proteus mirabilis & 1 \\
\hline
\end{tabular}

En Chile, si bien es probable que existan programas de vigilancia como política interna en algunos hospitales, sólo existe un artículo que aborda el tema de cultivos en cirugía de urgencia. En dicho estudio se evaluó un grupo seleccionado de pacientes operados por hernia inguinal estrangulada, sin necrosis intestinal ni peritonitis. En este grupo seleccionado de pacientes la tasa de cultivos positivos fue muy baja, identificándose en su mayoría bacterias de origen cutáneo ${ }^{13}$. A nivel latinoamericano, en Colombia, se realizó un estudio similar al nuestro con una tasa de cultivos mayor $(70,8 \%)$, pero con gérmenes similares ${ }^{14}$. Los datos de este trabajo constituyen la primera experiencia y resultados de la realidad local en un Hospital Universitario respecto al perfil microbiológico de los pacientes que consultan con patología quirúrgica de urgencia. En nuestros datos la tasa de positividad fue bastante menor, para lo que existen diversas posibles explicaciones. Realizar más de una muestra para análisis, el uso de más técnicas de cultivo; así como incorporar algunos datos del intraoperatorio no evaluados en este estudio, como presencia de líquido libre y sus características, grado de contaminación, podría permitir identificar en ciertos grupos de pacientes una tasa más alta de positividad. Si bien que $E$. coli sea el microorganismo más frecuentemente aislado $(66,1 \%$ de los cultivos positivos) no es una sorpresa, el análisis de susceptibilidad resulta interesante. Destaca su buena susceptibilidad para aminoglicósidos y carbapenémicos y su menor susceptibilidad para cefalosporinas de $1^{\mathrm{a}}$ y $2^{\mathrm{a}}$ generación. La baja sensibilidad a Ampicilina/Sulbactam es concordante con la literatura internacional, que reporta sensibilidades incluso más bajas $(<50 \%)$, así como también la menor sensibilidad a fluoroquinolonas ${ }^{12}$. En relación a $K$. pneumoniae, llama la atención la excelente susceptibilidad para cefalosporinas y carbapenémicos. 
Sin embargo, se repite una menor susceptibilidad para Ampicilina/Sulbactam, al igual que E. coli. Ninguna de las cepas de E. coli ni K. pneumoniae fue clasificada como productora de betalactamasa de espectro extendido (BLEE). Para el caso de gérmenes no fermentadores como P. aeruginosa, se evalúan susceptibilidades frente a carbapenémicos, cefalosporinas de $3^{\text {a }}$ generación, aminoglicósidos y quinolonas, observando altos porcentajes de susceptibilidad con todos los esquemas in vitro.

En conclusión, los microorganismos más frecuentemente aislados corresponden a enterobacterias, en especial E. coli. El perfil de resistencia manifestado por las bacterias más frecuentemente aisladas es similar al perfil internacional, aun cuando los porcentajes de resistencia son bastante más bajos. Se debe tomar en cuenta al momento del inicio de terapia empírica, que las infecciones intraabdominales suelen ser polimicrobianas. Por tanto, el blanco principal de la terapia antibiótica empírica iniciada previa a la cirugía, debe ser concordante a la susceptibilidad de éstas a los antibióticos disponibles; ajustándose una vez identificado el germen y antibiograma de los cultivos. Cabe comentar al margen, que los resultados de esta vigilancia microbiológica han llevado a cambios en los esquemas actualmente utilizados en nuestra institución; mostrando así, que llevar a cabo programas de esta categoría tiene un impacto directo en la calidad de atención a nuestros pacientes. Como política local se iniciará un programa de vigilancia periódica, incluyendo en próximos estudios una mayor cantidad de variables que permitan un análisis más acabado del aspecto local de resistencia a antimicrobianos, como por ejemplo, tiempo de evolución y tipo de herida quirúrgica. Asimismo, es fundamental recalcar el rol del acto quirúrgico como pilar fundamental en el tratamiento de estos pacientes.

\section{Responsabilidades éticas}

Protección de personas y animales. Los autores declaran que para esta investigación no se han realizado experimentos en seres humanos ni en animales.

Confidencialidad de los datos. Los autores declaran que en este artículo no aparecen datos de pacientes.

Derecho a la privacidad y consentimiento informado. Los autores declaran que en este artículo no aparecen datos de pacientes.

\section{Financiación} ción.

El presente trabajo no posee fuente de financia-

\section{Conflicto de intereses}

Los autores declaran no tener ningún conflicto de intereses.

\section{Bibliografía}

1. Martens E, Demain AL. The antibiotic resistance crisis, with a focus on the United States. J Antibiot (Tokyo). 2017 Mar 1. doi: 10.1038/ja.2017.30. [Epub ahead of print].

2. Silva OF, Cifuentes DM, Pinto CME. Resultados de la vigilancia de susceptibilidad antimicrobiana en Chile: Consolidando una red. Rev Chil Infectol. 2011;28:19-27.

3. Hackel MA, Badal RE, Bouchillon SK, Biedenbach DJ, Hoban DJ. Resistance Rates of Intra-Abdominal Isolates from Intensive Care Units and Non-Intensive Care Units in the United States: The Study for Monitoring Antimicrobial Resistance Trends 2010-2012. Surg Infect (Larchmt). 2015;16:298-304.
4. Morrissey I, Hackel M, Badal R, Bouchillon S, Hawser S, Biedenbach DA Review of Ten Years of the Study for Monitoring Antimicrobial Resistance Trends (SMART) from 2002 to 2011. Pharmaceuticals (Basel). 2013;6: 1335-46.

5. Hindler JF, Stelling J. Analysis and presentation of cumulative antibiograms: a new consensus guideline from the Clinical and Laboratory Standards Institute. Clin Infect Dis. 2007;44:867-73.

6. García-Sánchez JE, García-García MI, García-Garrote F, Sánchez-Romero I. Diagnóstico microbiológico de las infecciones intraabdominales. Enferm Infecc Microbiol Clin. 2013;31:230-9.

7. Fraser A, Paul M, Almanasreh N, Tacconelli E, Frank U, Cauda R, et al. TREAT Study Group. Benefit of appropriate empirical antibiotic treatment: thirty-day mortality and duration of hospital stay. Am J Med. 2006;119:970-6.

8. Jean SS, Coombs G, Ling T, Balaji V, Rodrigues C, Mikamo H, et al. Epidemiology and antimicrobial susceptibility profiles of pathogens causing urinary tract infections in the Asia-Pacific region: Results from the Study for Monitoring Antimicrobial Resistance Trends (SMART), 2010-2013. Int J Antimicrob Agents 2016;47:328-34.

9. Jean SS, Hsueh PR; SMART Asia-Pacific Group. Distribution of ESBLs, AmpC $\beta$-lactamases and carbapenemases among Enterobacteriaceae isolates causing intraabdominal and urinary tract infections in the Asia-Pacific region during 2008-14: results from the Study for Monitoring Antimicrobial Resistance Trends 


\section{ARTíCULO DE INVESTIGACIÓN}

(SMART). J Antimicrob Chemother. 2017;72:166-71.

10. Karlowsky JA, Lob SH, Kazmierczak KM, Badal RE, Young K, Motyl MR, et al. In Vitro Activity of Imipenem against Carbapenemase-Positive Enterobacteriaceae: SMART Global Surveillance Program 2008-2014. J Clin Microbiol. 2017 Mar 15. pii: JCM.02316-16. doi: 10.1128/JCM.0231616. [Epub ahead of print].

11. Lob SH, Hackel MA, Kazmierczak KM, Hoban DJ, Young K, Motyl MR, et al. In vitro activity of imipenem-relebactam against gram-negative bacilli isolated from patients with lower respiratory tract infections in the United States in 2015 - Results from the SMART global surveillance program. Diagn Microbiol Infect Dis. 2017 Mar 2. pii: S0732-8893(17)30074-3. doi: 10.1016/j. diagmicrobio.2017.02.018. [Epub ahead of print].

12. Zalacain M, Biedenbach DJ, Badal RE, Young K, Motyl M, Sahm DF.

Pathogen Prevalence and Antimicrobial Susceptibility Among Enterobacteriaceae Causing Hospital-associated Intraabdominal Infections in Adults in the United States (2012-2013). Clin Ther.
2016;38:1510-21. doi: 10.1016/j. clinthera.2016.04.035. Epub 2016 May 24.

13. Morales J, Canales L, Morales C, Rebolledo R, Novoa J, Otero E. Perfil microbiológico del saco herniario en hernias complicadas operadas en el Servicio de Urgencias. Rev Chil Cir. 2009;61:448-52.

14. Vallejo MA, Cuesta D, Flórez L, Correa A, Llanos C, Isaza B, et al. Características clínicas y microbiológicas de la infección intraabdominal complicada en Colombia: un estudio multicéntrico. Rev Chil Infectol. 2016; 33:261-7. 